\title{
THE TOPOLOGICAL DEGREE FOR NONCOMPACT NONLINEAR MAPPINGS IN BANACH SPACES
}

\author{
BY FELIX E. BROWDER AND ROGER D. NUSSBAUM
}

Communicated by Felix Browder, December 26, 1967

Let $X$ and $Y$ be Banach spaces, $G$ an open subset of $X$. If we denote the closure of $G$ by $\operatorname{cl}(G)$, let $f$ be a mapping of $\operatorname{cl}(G)$ into $Y$.

For $X=Y$ and $f$ a compact mapping, Leray and Schauder [9] gave a definition of topological degree for mappings of the form $I-f$ on the open set $G$ over a point $a$ of $X$ whenever $(I-f)^{-1}(a)$ is a compact subset of $G$. The Leray-Schauder degree for compact displacements is the most subtle tool of the classical fixed point theory of compact mappings, and if one proposes to carry through an extension of the fixed point and mapping theory to more general mappings which are neither of the form $f$ or $I-f$, with $f$ compact, an important step in such a program is to find a more general framework for the concept of the topological degree. In particular, it would be desirable to bring within such a framework the various classes of nonlinear noncompact operators for which fixed point and mapping results have been obtained in recent years: the asymptotically compact mappings, the nonexpansive and semi-contractive mappings of uniformly convex Banach spaces $X$ into themselves, the monotone and semimonotone mappings of a reflexive Banach space $X$ into its conjugate space $X^{*}$, the accretive and semi-accretive mappings of a Banach space $X$ into itself, and their more general analogues (cf. [1], [2], [3], [4]).

It is the object of the present note to present such a framework for a class of mappings which extends that considered by one of the writers in [1]. We give below, by successive stages, the definition of the generalized degree and establish its basic properties.

Definition 1. Let $X$ be a Banach space, $G$ an open subset of $X, T a$ continuous mapping of $\operatorname{cl}(G)$ into $X$ such that $I-T$ is locally compact (i.e. each point of $\mathrm{cl}(G)$ has a neighborhood $N$ such that $I-T(N)$ is relatively compact in $X)$ and such that $T^{-1}(a)$ is compact for a given element $a$ of $X$. Then we define $\operatorname{deg}(T, G, a)$, the degree of $T$ on $G$ over $a$ to be $\operatorname{deg}_{L S}(T, V, a)$, the Leray-Schauder degree of $T$ over $a$ on any open neighborhood $V$ of $T_{-}{ }^{1}(a)$ such that $(I-T)(V)$ is relatively compact in $X$.

Lemma 1. Let $T$ be a mapping satisfying the conditions of Definition 1. Then:

(a) $\operatorname{deg}(T, V, a)$ is well-defined by Definition 1 for any a such that $T^{-1}(a)$ is a compact subset of $G$. 
(b) The degree is additive in the usual sense, i.e. if $G_{1}$ and $G_{2}$ are open subsets of $G$ such that $T^{-1}(a)$ has no points in common with (cl(G) $\left.-\left(G_{1} \cup G_{2}\right)\right) \cup\left(G_{1} \cap G_{2}\right)$, then

$$
\operatorname{deg}(T, G, a)=\operatorname{deg}\left(T, G_{1}, a\right)+\operatorname{deg}\left(T, G_{2}, a\right) .
$$

(c) If $\operatorname{deg}(T, G, a) \neq 0$, then $T$ has an a-point in $G$, i.e. there exists some $x_{0}$ in $G$ such that $T\left(x_{0}\right)=a$.

(d) If $\Omega$ is an open subset of $X \times[0,1], \Omega_{t}$ its slice at parameter value $t, T$ a mapping of $\mathrm{cl}(\Omega)$ into $X$ such that $\pi-T$ is locally compact, $(\pi(x, t)=x)$, and such that for a given a in $X, T^{-1}(a)$ is a compact subset of $\Omega$, then (setting $T_{t}$ to be the induced mapping of $\Omega_{t}$ into $\left.X\right) \operatorname{deg}\left(T_{t}, \Omega_{t}, a\right.$ ) is well-defined for all $t$ in $[0,1]$ and independent of $t$.

Proof of Lemma 1. This is an easy direct translation of the corresponding properties of the Leray-Schauder degree (Leray-Schauder [9], Nagumo [10], Cronin [7]).

Definition 2. Let $X$ and $Y$ be real Banach spaces, $G$ an open subset of $X, S$ a mapping of $\operatorname{cl}(G) \times \operatorname{cl}(G)$ into $Y$ such that the following conditions hold:

(a) For each fixed $v$ in $\operatorname{cl}(G), S_{v}=S(\cdot, v)$ is a homeomorphism of $G$ on an open subset $G_{v}$ of $Y$, with $S_{v}$ mapping $\operatorname{cl}(G)$ homeomorphically onto $\operatorname{cl}\left(G_{v}\right)$.

(b) The mapping $v \rightarrow S_{v}$ is a locally compact mapping of $\operatorname{cl}(G)$ into the space of homeomorphisms of $\mathrm{cl}(G)$ into $Y$, with the topology of uniform convergence on $\mathrm{cl}(G)$.

Let $T$ be the mapping of $\operatorname{cl}(G)$ into $Y$ given by $T(u)=S(u, u)$, for $u$ in $\operatorname{cl}(G)$. We suppose that for a given element a of $Y, T^{-1}(a)$ is a compact subset of $G$. Then we apply the following procedure to obtain a concept of degree for the pair $[T, S]$, (i.e. $T$ in the representation $S$ ):

(1) Let $G_{1}$ be the subset of those $v$ in $G$ such that the equation $S(u, v)=a$ has a solution $u$ in $G$. Let $C$ be the mapping of $G_{1}$ into $X$ given by $C(v)=u$, where $u$ is this solution, and suppose that $G_{1}$ is open and that $C$ can be extended to a mapping of $\operatorname{cl}\left(G_{1}\right)$ into $X$. Then:

(2) We set

$$
\operatorname{deg}([T, S], G, a)=\operatorname{deg}\left(I-C, G_{1}, 0\right) .
$$

Theorem 1. Let $X$ and $Y$ be Banach spaces, $G$ a connected open subset of $X, S$ a mapping of $\operatorname{cl}(G) \times \operatorname{cl}(G)$ into $Y$ which satisfies the conditions (a) and (b) of Definition 2. Let $T(u)=S(u, u)$ for $u$ in $\mathrm{cl}(G)$, and suppose that $T^{-1}(a)$ is a compact subset of $G$ for a given element $a$ of $Y$. Then: 
(i) The set $G_{1}=\{v \mid v \in G$, there exists $u$ in $G$ such that $S(u, v)=a\}$ is an open subset of $G$ and hence of $X$.

(ii) The set $G^{+}=\{v \mid v \in \operatorname{cl}(G)$, there exists $u$ in $\operatorname{cl}(G)$ such that $S(u, v)=a\}$ contains the closure of $G_{1}$. The mapping $C^{+}$of $G_{1}^{+}$into $X$ given by $C^{+}(v)=u$, with $u$ as above, is a locally compact mapping of $\operatorname{cl}\left(G_{1}\right)$ into $X$ and its fixed point set is a compact subset of $G_{1} . C^{+}$has $C$ as its restriction to $G_{1}$, and maps bdry $\left(G_{1}\right)$ into bdry $(G)$.

(iii) $\operatorname{deg}([T, S], G, a)$ is thus well-defined by the steps (1) and (2).

Proof of Theorem 1. Suppose that $v$ lies in $G_{1}$. Then $a$ lies in $S_{v}(G)$, and there exists a ball about $a$ which does not intersect $S_{v}$ (bdry $G$ ). If $v_{1}$ is sufficiently close to $v$, there exists a ball about $a$ which does not intersect $S_{v_{1}}$ (bdry $G$ ), and we may assume that $S_{v_{1}}(u)$ also lies in this ball. On the other hand, since $S_{v_{1}}$ is a homeomorphism of $G$ on $G_{v_{1}}$ which carries bdry $(G)$ onto bdry $\left(G_{v_{1}}\right)$, it follows that $G_{v_{1}}$ coincides with one component of $X-S_{v_{1}}$ (bdry $G$ ). Hence, the whole ball about $a$ lies in $G_{v_{1}}$, i.e. in particular $a$ lies in $S_{v_{1}}(G)$ and $v_{1}$ lies in $G_{1}$. Hence $G_{1}$ is open in $X$.

If $v$ lies in $\operatorname{cl}\left(G_{1}\right)$, there exists a sequence $\left\{v_{k}\right\}$ in $G_{1}$ converging to $v$. In particular, $S_{v_{k}}$ converges to $S_{v}$. Let $S\left(u_{k}, v_{k}\right)=a$. Then $S\left(u_{k}, v\right)$ $=a+\left[S\left(u_{k}, v\right)-S\left(u_{k}, v_{k}\right)\right] \rightarrow a$. Since $S_{v}$ is a homeomorphism of $\operatorname{cl}(G)$ on $\operatorname{cl}\left(G_{v}\right)$, it follows that $a$ lies in $\operatorname{cl}\left(G_{v}\right)$, i.e. $v$ lies in $G_{1}^{+}$. The mapping $C^{+}$thus is defined on $\operatorname{cl}\left(G_{1}\right)$ and maps $\operatorname{cl}\left(G_{1}\right)$ into $\operatorname{cl}(G)$, and if $C^{+}(v)$ lies in $G, v$ must lie in $G_{1}$. Hence $C^{+}$maps the boundary of $G_{1}$ into the boundary of $G$. If $v$ is a fixed point of $C^{+}$, then $S(v, v)=a$, and $v$ lies in $T^{-1}(a)$ so that $v$ lies in $G$ and hence in $G_{1}$. Conversely every point of $T^{-1}(a)$ is a fixed point of $C^{+}$, and hence the fixed point set of $C^{+}$is a compact subset of $G_{1}$. Finally, if $v$ is a point of $\operatorname{cl}\left(G_{1}\right)$, there exists a neighborhood $N$ of $v$ such that the map $v \rightarrow S_{v}$ carries $N$ into a relatively compact set of homeomorphisms of $G$ into $Y$. Let $\left\{v_{k}\right\}$ be an infinite sequence in $N$. Then by passing to an infinite subsequence, we may assume that $S_{v_{k}} \rightarrow S^{\prime}$, where $S^{\prime}$ is a homeomorphism of $\operatorname{cl}(G)$ into $Y$. If $u_{k}=S_{v_{k}}{ }^{-1}(a)$, then $S^{\prime}\left(u_{k}\right) \rightarrow a$, so that $u_{k} \rightarrow\left(S^{\prime}\right)^{-1}(a)$. Then $C^{+}\left(v_{k}\right)=u_{k}$ converges and $C^{+}$is locally compact.

Q.E.D.

In the special case, when $S(u, v)=h(u)+f(v)$, with $h$ a homeomorphism of $\operatorname{cl}(G)$ into $Y$ and $f$ a locally compact mapping of $\operatorname{cl}(G)$ into $Y$, we identify $\operatorname{deg}([T, S], G, a)$ with $\operatorname{deg}\left(I-C, G_{1}, 0\right)$ where $C(v)$ $=h^{-1}(a-f(v)), G_{1}=f^{-1}(a-h(G))$. For the even more special case where $h=I-U$ with $U$ a strict contraction and $f$ of finite dimension, our definition coincides with definitions of the degree applied by Cacciopoli [5], Cronin [7], and Cesari [6]. For the slightly more gen- 
eral case in which $h=I-U, U$ strictly contractive, and $f$ is compact, it is related to a definition given in a recent note of Frum-Ketkov [8].

Theorem 2. For the degree $\operatorname{deg}([T, S], G, a)$, as defined above, we have the properties corresponding to those given in Lemma 1:

(i) The degree is additive.

(ii) If $\operatorname{deg}([T, S], G, a) \neq 0$, then $T^{-1}(a)$ is nonempty.

(iii) Let $\Omega$ be an open subset of $X \times[0,1], \Omega_{t}$ its slice at t, T a mapping of $\operatorname{cl}(\Omega)$ into $Y$ such that for a given element a of $Y, T^{-1}(a)$ is a compact subset of $\Omega$. For each $t$ in $[0,1]$, let $S_{t}$ be a mapping of $\operatorname{cl}\left(\Omega_{t}\right) \times \operatorname{cl}\left(\Omega_{t}\right)$ into $Y$ such that $T_{t}(u)=S_{t}(u, u)$ for all $u$ in $\mathrm{cl}\left(\Omega_{t}\right)$, with $S_{t}$ satisfying the conditions (a) and (b) of Definition 2 for $S$. Suppose that for each $(v, t)$ in $\Omega$, there is a neighborhood $N_{v} \subset \Omega_{t}$ and an interval $J$ such that $N_{v} \times J \subset \Omega$ and the map $\left.(w, s) \rightarrow S_{s}(\cdot, w)\right|_{N_{v}}$ is locally compact and continuous on $N_{v} \times J$. Then $\operatorname{deg}\left(\left[T_{t}, S_{t}\right], \Omega_{t}, a\right)$ is well-defined and independent of $t$ for $t$ in $[0,1]$.

Proof of Theorem 2. Properties (i) and (ii) follow trivially from the definition of $\operatorname{deg}([T, S], G, a)$. We need only prove property (iii). For each $t$ in $[0,1]$, the domain $\operatorname{cl}\left(\Omega_{t}\right)$ of $T_{t}$ may be different. Since $T^{-1}(a)$ is compact, we may cover it by a finite number of product neighborhoods in $\Omega$ of the form $N_{j} \times\left(t_{j}, t_{j+1}\right)$. When two such neighborhoods overlap, we may assume that for any common value of $t$, $\left(N_{j}-N_{k}\right) \times\{t\}$ does not intersect $T_{t}^{-1}(a)$. Hence $\operatorname{deg}\left(\left[T_{t}, S_{t}\right], N_{j}, a\right)$ $=\operatorname{deg}\left([T, S], N_{k}, a\right)$, and we may assume without loss of generality that $\Omega=N \times[0,1]$ and that $S_{t}$ is uniformly continuous from $[0,1]$ to the space of mapping of $N \times N$ to $Y$.

Let $G_{1, t}=\left\{v \mid a\right.$ lies in $\left.S_{t, v}(N)\right\}$. By the same argument as we used in the proof of Theorem $1, \Omega_{1}=U_{t \in[0,1]} G_{1, t} \times\{t\}$ is open in $X \times[0,1]$ and the mapping $C_{t}^{+}$is uniformly continuous in $t$. Applying Lemma 1 , we have $\operatorname{deg}\left(I-C_{t}^{+}, G_{1, t}, 0\right)$ is independent of $t$. Since $\operatorname{deg}\left(\left[T_{t}, S_{t}\right], \Omega_{t}, a\right)$ $=\operatorname{deg}\left(I-C_{t}^{+}, G_{1, t}, 0\right)$, the conclusion of Theorem 2 follows. Q.E.D.

If we wish to make the degree we have defined depend only on the mapping $T$ and not on the representation $S$, we can do so by restricting the homeomorphisms $S_{v}$ to lie in a convex class, as follows:

THEOREM 3. Let $T$ be a mapping of $\operatorname{cl}(G)$ into $Y$, with $T(u)=S(u, u)$ for a mapping $S$ of $\mathrm{cl}(G) \times \mathrm{cl}(G)$ into $Y$ satisfying the conditions (a) and (b) of Definition 2. Suppose that $T^{-1}(a)$ is compact for a given element a of $Y$, and that there exists a convex set $H_{0}$ of homeomorphisms of $\operatorname{cl}(G)$ into $Y$ such that $S_{v}$ lies in $H_{0}$ for each $v$ in $\operatorname{cl}(G)$. Then:

(a) For any pair of representations, $S_{0}$ and $S_{1}$ satisfying this condition for a given $H_{0}$ for the same mapping $T$, 


$$
\operatorname{deg}\left(\left[T, S_{0}\right], G, a\right)=\operatorname{deg}\left(\left[T, S_{1}\right], G, a\right) .
$$

(b) If, in addition, the mapping $v \rightarrow S_{v}$ is compact from $\mathrm{cl}(G)$ to the homeomorphisms of $\operatorname{cl}(G)$ into $Y$, then $\operatorname{deg}([T, S], G, a)=\operatorname{deg}(T, G, a)$ is invariant under permissible homotopies, i.e. under homotopies of $T_{t}$ on open sets $\Omega_{t}$ in $X$ such that $\bigcup_{t \in[0,1]} \Omega_{t} \times\{t\}$ is open and $T_{t}(u) \neq a$ on the boundary of $\Omega_{t}$.

Proof of Theorem 3. Proof of (a). We consider deg $\left(\left[T, S_{t}\right], G, a\right)$ where $S_{t}=(1-t) S_{0}+t S_{1}$. This is independent of $t$ by property (c) of Theorem 2 .

Proof OF (b). If the mapping $v \rightarrow S_{v}$ is compact, then the condition that $T^{-1}(a)$ is a compact subset of $G$ is equivalent to the condition that $T^{-1}(a)$ does not intersect the boundary of $G$. Let $\left\{T_{t}\right\}$ be a permissible homotopy. We may assume without loss of generality that $\Omega=G \times[0,1]$, and by splitting up the interval $[0,1]$ into small subintervals, we may assume that $T_{0}(u)$ and $T_{1}(u)$ are at most $\epsilon$ apart for $u$ on the boundary of $G$ and that $\left\|T_{0}(u)-a\right\|>\epsilon$ for all $u$ in bdry $(G)$. Let $S_{0}$ and $S_{1}$ be representations of $T_{0}$ and $T_{1}$, respectively. Then $S_{t}=(1-t) S_{0}+t S_{1}$ is a permissible homotopy of representations in the sense of Theorem 2(c).

Q.E.D.

We note that a number of the most interesting classes of homeomorphisms such as $h=I-U$ with $U$ a strict contraction, $h$ a strongly monotone mapping, or $h$ a strongly accretive mapping, etc. are closed convex sets in the sense of Theorem 3 . As an illustrative corollary of Theorems 2 and 3, we consider the following simple result:

Theorem 4. Let $G$ be an open convex subset of a Banach space $X$, $0 \in G, U$ a strictly contractive map of $\operatorname{cl}(G)$ into $X$, (i.e. $\|U u-U v\|$ $\leqq k\|u-v\|$ with $k<1), R$ a locally compact mapping of $\operatorname{cl}(G)$ into $X$. Suppose that for each $t \geqq 1, t I-(U+R)$ has a compact set of zeroes in $\operatorname{cl}(G)$ which does not intersect the boundary of $G$. Then $U+R$ has a fixed point in $G$.

Proof of Theorem 4. Apply Theorem 2 to $S_{t}(u, v)=(I-t U) u$ $-t R v$.

As the final stage of our note, we consider the limit case of those mappings $T$ which are limits of those represented in Definition 2.

Theorem 5. Let $X$ and $Y$ be Banach spaces, $T$ a mapping of $\mathrm{cl}(G)$ into $Y$ where $G$ is an open subset of $G$. Let $H_{0}$ be a convex family of homeomorphisms of $\mathrm{cl}(G)$ into $Y$. Suppose that there exists a sequence of mappings $\left\{T_{k}\right\}$ converging uniformly to $T$ on $\operatorname{cl}(G)$ with each $T_{k}$ of the form $T_{k}(u)=S_{k}(u, u)$ where $S_{k}$ is a mapping of $\operatorname{cl}(G) \times \mathrm{cl}(G)$ into $Y$ 
satisfying the conditions of Definition 2 with each $S_{k, v}$ lying in $H_{0}$ and with the mappings $v \rightarrow S_{k, v}$ compact on $\operatorname{cl}(G)$. Then:

(a) $\lim _{k} \operatorname{deg}\left(\left[T_{k}, S_{k}\right], G, a\right)$ exists and may be denoted by $\operatorname{deg}([T, S]$, $G, a)$, provided that there exists $\epsilon>0$ such that for $u$ on bdry $G$, $\|T(u)-a\| \geqq \epsilon$.

(b) If $\operatorname{deg}([T, S], G, a) \neq 0$, then a lies in $\operatorname{cl}(T(G))$. In particular, if $T(\mathrm{cl}(G))$ is closed, then a lies in $T(G)$.

(c) $\operatorname{deg}\left(\left[T_{t}, S_{t}\right], \Omega_{t}, a\right)$ is invariant under homotopies provided that $\operatorname{dist}(T$ (bdry $\Omega$ ), $a)>0$.

The proof of Theorem 5 will appear elsewhere. The restriction that the homeomorphisms $S_{k, v}$ lie in the convex set $H_{0}$ is not necessary if one assumes the $S_{k}$ are defined on $X \times X$ and converge uniformly on bounded subsets of $X \times X([1])$.

\section{BIBLIOGRAPHY}

1. F. E. Browder, Nonlinear operators in Banach spaces, Math. Ann. 162 (1966), 280-283.

2. - Fixed point theorems for nonlinear semicontractive mappings in Banach spaces, Arch. Rational Mech. Anal. 21 (1966), 259-269.

3. - Semicontractive and semiaccretive nonlinear mappings in Banach spaces, Bull. Amer. Math. Soc. 74 (1968), 660-665.

4. - Fixed point and mapping theorems in infinite dimensional spaces, Proc. Conference on Inf. Dim. Topology, 1967 (to appear).

5. R. Cacciopoli, Sulle corrispondenze funzionali inverse diramate, Rend. Accad. Naz. Lincei 24 (1936), 258-263.

6. L. Cesari, Functional analysis and periodic solutions of nonlinear differential equations, Contributions to Differential Equations 1 (1963), 149-187.

7. J. Cronin, Fixed points and topological degree in nonlinear analy'sis, Math. Surveys No. 11, Amer. Math. Soc., Providence, R. I., 1964.

8. R. L. Frum-Ketkov, On mappings of the sphere of a Banach space, Dokl. Akad. Nauk SSSR 175 (1967), 1229-1231 = Soviet Math. Dokl. 8 (1967), 1004-1006.

9. J. Leray and J. Schauder, Topologie et equations fonctionelles, Ann. Sci. Ecole Norm Sup. 51 (1934), 45-78.

10. M. Nagumo, Degree of mapping in convex linear topological spaces, Amer. J. Math. 73 (1951), 497-511.

University of Chicago 